\title{
Vinculação aos pais e relação amorosa: Papel mediador dos conflitos interparentais em jovens adultos
}

\author{
Catarina Pinheiro Mota ${ }^{1}$ \& Carine Martins ${ }^{2}$ \\ ${ }^{1}$ Universidade de Trás-os-Montes e Alto Douro - UTAD, Vila Real Portugal/ Centro de Psicologia da \\ Universidade do Porto, Porto, Portugal \\ ${ }_{2}^{2}$ Universidade de Trás-os-Montes e Alto Douro - UTAD, Vila Real, Portugal
}

\begin{abstract}
Resumo: A qualidade das relações estabelecidas precocemente com os pais mostra-se de extrema relevância no desenvolvimento emocional e social dos jovens. A construção e desenvolvimento das relações de bases seguras sugere maior disponibilidade para o estabelecimento de relações fora do seio familiar, nomeadamente com o par amoroso, estando as vivências afetivas inseguras associadas a dificuldades no estabelecimento e manutenção de relações amorosas. 0 presente estudo teve como objetivo analisar a qualidade de vinculação aos pais e testar o seu efeito na vinculação amorosa em adultos emergentes, assim como testar o papel mediador dos conflitos interparentais na associação anterior. Participaram 505 indivíduos com idades compreendidas entre os 18 e os 25 anos de idade $(M=$ 20.59; $D P=1.78)$, que responderam aos Questionários: de Vinculação ao Pai e à Mãe (QVPM), de Vinculação Amorosa (QVA), e ao The Children's Perception of Interparental Conflict Scale (CPIC). Os resultados apontam para um efeito preditor positivo da qualidade de laço emocional aos pais face à qualidade de vinculação ao par amoroso. Verifica-se, ainda, que a perceção de conflitos interparentais exerce um papel mediador na associação entre a vinculação aos pais e a vinculação amorosa.
\end{abstract}

\section{Palavras-chave: Vinculação aos pais; vinculação amorosa; conflitos interparentais.}

Attachment to parents and romantic relationship: Mediational role of interparental conflicts in young adults: The quality of early relationships established with the parents seems to be extremely relevant in the physical and emotional development of young people. The construction and development of secure bases relationships suggest greater willingness to establish relationships outside the family environment, in particular the romantic relationship. However, insecure affective experiences with primary figures of affection are associated with difficulties in external relations. This study aims to analyse the attachment to parents and test its effect on romantic relationship in emerging adults, as well as test the mediating role of interparental conflict in previous association. Were participating 505 individuals, aged between 18 and 25 years old $(M=20.59 ; D P=1.78)$, that answer to Attachment Questionnaire to Father and Mother (QVPM), Romantic Attachment Questionnaire (QVA), and to Children`s Perception of Interparental Conflict Scale (CPIC). The results point to a positive predictor effect of attachment to parents on romantic attachment. It is observed also that the interparental conflicts have a mediator role in the association between attachment to parents and the romantic attachment.

Keywords: Attachment to parents; attachment to romantic peer; interparental conflict.

Descrita originalmente por Bowlby (1969), a vinculação traduz a necessidade inata do ser humano para criar e estabelecer laços afetivos de grande intensidade com as figuras cuidadoras, possibilitando o desenvolvimento e crescimento emocional do indivíduo. Existe no ser humano um sistema de controlo mediado por comportamentos instintivos. Tais comportamentos de procura de proximidade destinam-se a manter ou atingir a proximidade desejada das figuras significativas de afeto, geralmente os pais, com o objetivo de obter apoio, proteção e segurança. As experiências relacionais com estas figuras vão criando uma organização interna que promove o desenvolvimento de modelos internos dinâmicos. Estes modelos integram mapas cognitivos, sentimentos, crenças, expectativas, basicamente representações que a criança constrói e tem sobre si própria e o seu comportamento, bem como dos outros e do mundo, e cuja complexificação tende a aumentar ao longo da vida (Bowlby, 1988). Uma característica particular é a capacidade de reorganização dos modelos e de adaptação às características dos novos períodos de desenvolvimento e contextos ao longo do ciclo de vida. Os modelos internos dinâmicos tornam-se mais

${ }^{1}$ Morada para correspondência: Catarina Pinheiro Mota, Departamento de Educação e Psicologia da UTAD, Quinta de Prados, 5000-801, Vila Real. E-mail: catppmota@utad.pt. Esta investigação é parcialmente suportada pela FCT através do projeto PEst-C/PSI/UI0050/2011 e o FEDER com fundos do programa COMPETE sob o projeto FCOMP-01-0124-FEDER-022714. 
positivos sob o ponto de vista da adaptação à medida que a procura de proximidade da criança vai sendo correspondida de forma satisfatória pelas figuras cuidadoras primárias, facilitando o desenvolvimento de uma base segura. 0 estabelecimento destes laços irá funcionar como fator protetor face a situações de risco, e como base segura para a criação de uma imagem positiva de si e dos outros, bem como a exploração do meio circundante (Ainsworth, 1969; Bowlby, 1969). Deste modo, Bowlby (1969) aponta que a qualidade das relações de vinculação, ainda que não de forma determinante, podem servir de modelo para a construção de relações futuras ao longo da vida. Em função dos cuidados que lhe são transmitidos, poderá evidenciar uma crescente maturidade emocional e, assim, uma estrutura emocional mais capaz para enfrentar vicissitudes. Todavia, uma criança com relações inseguras tende a avaliar o mundo como um local perigoso, mediante a representação de si como incapaz para enfrentar as dificuldades, e dos outros como pouco competentes para satisfazer as suas necessidades (e.g. Bretherton, 2000). De acordo com a evidência empírica, os padrões de vinculação que se estabelecem durante os primeiros anos de vida podem ser significativos na vulnerabilidade face a adversidades internas e externas ao longo do desenvolvimento (e.g. Feeney, Cassidy, \& Ramos-Marcuse, 2008; Zimmermann, 2004). Nesta medida, indivíduos que mantêm relações saudáveis e positivas com as figuras parentais ou outras figuras primordiais de afeto tendem a ser menos vulneráveis face a situações de risco, quando comparados com aqueles que apresentam dificuldades nas suas relações, nomeadamente falta de afeto, insegurança e rejeição.

$\mathrm{Na}$ adolescência e emergência da adultícia a internalização de novas competências cognitivas abrem a possibilidade de pensar sobre as relações no abstrato - a reorganização de modelos de base segura dos pais surge como um dos facilitadores no processo de progressiva diferenciação self-outro. A separação sugere um passo relevante no desenvolvimento dos jovens, constituindo-se num preditor do alargamento da rede de apoio emocional. Ela não pressupõe uma rutura com o contexto emocionalmente significativo da relação parental. Ao contrário, é no seio de relações onde é permitido aos jovens que se explorem a si próprios e ao mundo, mesmo que no desacordo, que se mantém a base segura, alargando potencialmente o sistema de vinculação a figuras alternativas de segurança (e.g. Rocha, Mota \& Matos, 2011). A relação com o par amoroso desempenha para os jovens um ensaio maior para futuras vivências românticas emocionalmente investidas. Ao potenciarem uma maior perceção de si e da dinâmica de si com o outro, permitem o desenvolvimento de capacidades de tolerância e respeito pelo espaço vital do outro. 0 percurso de afastamento/proximidade com cada uma destas figuras representa um cenário de progressiva inserção num contexto. A procura de proximidade não é obrigatoriamente física, no sentido da inclusão do sistema sexual na relação, mas implica uma ânsia de intimidade partilhada, sobretudo no final da adolescência e início da emergência da adultícia (Meeus, Iedema, Maassen, \& Engels, 2005; Mikulincer, Gillath, \& Shaver, 2002).

Assim, ao longo do desenvolvimento, jovens que percecionaram confiança e apoio nas figuras significativas de vinculação tendem a desenvolver personalidades estruturadas, caracterizadas por uma vivência emocional mais estável e confiante para enfrentar o futuro, bem como face ao estabelecimento e qualidade das novas relações românticas (Mikulincer et al., 2002; Morgan \& Shaver, 1999). Contrariamente, os adultos emergentes com estilos de vinculação insegura tendem a mostrar-se mais resistentes, defensivos ou retractivos face ao estabelecimento de relações de grande proximidade (Atger, 2004; Castillo, 2007; Feeney et al., 2008; Zimmermann, 2004). Na conceção de vários autores, uma vinculação segura às figuras parentais pode ser preditora do desenvolvimento saudável de relações românticas na vida adulta, sendo estas relações caracterizadas pela confiança, satisfação, compromisso, interdependência e suporte mútuo (e.g., Altin \& Terzi, 2010; Beato, 2008; Fraley, 2002; Matos, 2002; Pereira, Ferreira, \& Paredes, 2012; Waters \& Cummings, 2000). Estudos apontam para uma elevada correspondência entre as representações das figuras parentais e a escolhas do par romântico (e.g. Crowell, Treboux, \& Waters, 2002; Treboux, Crowell, \& Waters, 2004). Assim, embora as primeiras representações de vinculação sejam integradas e sentidas como cognições e crenças baseadas na experiência ao longo do tempo (Bretherton, 1985; Waters, Waters, \& Crowell, 2000), elas podem ser entendidas como um filtro para a compreensão da experiência e podem orientar a ação noutras relações efetivas ao longo da vida.

De uma forma geral, na adolescência o par amoroso poderá desempenhar a função de porto seguro, sendo caracterizado como uma figura afetivamente significativa à qual se recorre em situação de necessidade, procurando conforto, proteção, apoio e alívio (Ainsworth, 1991). No entanto, e à medida que as relações vão sendo pautadas por características como a duração ao longo do tempo, intensidade e estabilidade podem assumir a função de vinculação. Em especial, a partir da jovem adultícia a construção de relações mais estáveis e duradouras na relação com o par amoroso pode proporcionar sentimentos de segurança, na medida em que promove a exploração do mundo e gera um sentimento de competência pessoal (Shaver \& Hazan, 1988). Com base nesta premissa, o indivíduo irá sentir-se mais seguro ao 
percecionar o seu parceiro como acessível e responsivo, sendo que este poderá vir a desempenhar um papel de base segura nos momentos de exploração do meio e será uma fonte de segurança e proteção em momentos ameaçadores (Hazan \& Shaver, 1990).

Todavia, importa referir que existem alguns fatores passíveis de condicionar desenvolvimento da qualidade de vinculação com os pais, assim como no desenvolvimento de relações amorosas saudáveis. Entre esses fatores, encontra-se a perceção de conflitos interparentais, passíveis de influenciar reações e comportamentos posteriores na relação com o parceiro romântico (Beato, 2008; Garcia, Marín, \& Currea, 2006; Moura \& Matos, 2008; Moura, Santos, Rocha, \& Matos, 2010; Reese-Weber \& Kahn, 2005). De ressaltar que os episódios de conflitos interparentais resolvidos de forma construtiva não conferem necessariamente um desajuste emocional aos elementos da família. Ao invés disso, conflitos interparentais, com níveis de intensidades que não subestimem o bem-estar emocional dos intervenientes, podem inclusive contribuir para um amadurecimento emocional de todos os elementos da família, e particularmente dos jovens, já que é promovida a aprendizagem de discussões menos coercivas e agressivas (Cummings, 1998; Grych \& Fincham, 1990). De uma forma particular, alguns estudos realizados neste âmbito, cujo objetivo foi verificar o efeito dos conflitos interparentais nas relações românticas de jovens adultos, mostraram um impacto negativo do conflito interparental não resolvido na qualidade e manutenção de relações românticas (e.g., Cui \& Fincham, 2010; Cusimano \& Riggs, 2013; Feeney, 2006; Melo \& Mota, 2014; Moura \& Matos, 2008; Moura et al., 2010). Herzog e Coney (2002) sugerem que os padrões destrutivos de comportamentos observados no seio familiar podem ser reproduzidos mais tarde e interferir negativamente no bem-estar emocional do sujeito e do parceiro romântico, assim como na qualidade dos padrões comunicacionais estabelecidos. Beato (2008) reforça esta ideia, salientando que a perceção de frequentes e intensos conflitos por parte dos jovens leva a que estes assumam um padrão relacional inseguro que, consequentemente, se repercute nas relações românticas, podendo ocasionar menor confiança no parceiro amoroso. Por outro lado, a literatura tem vindo apontar que as diferentes respostas emocionais e comportamentais dos filhos à exposição de conflitos, depende da forma como os pais gerem e resolvem as suas divergências, das estratégias de resolução e dos padrões de conflito (e.g., Cummings, Goeke-Morey, \& Papp, 2004; Melo \& Mota, 2014; Mota \& Matos, 2009, 2014). O processo de resolução pode apresentar-se como algo capaz de facilitar o crescimento emocional dos indivíduos. Tomar contacto com a existência de dificuldades no seio parental, que devem ser debatidas e resolvidas no sentido de alcançar o bem-estar e ajustamento psicológico, pode ser relevante para a aprendizagem acerca de uma forma adaptativa de resolver conflitos em futuras relações (Benetti, 2006; Lindsey, Colwell, Frabutt, \& MacKinnon-Lewis, 2006; Mota \& Matos, 2011). 0 estudo realizado por Goeke-Morey, Cummings, e Papp (2007), com o objetivo de analisar as implicações dos conflitos interparentais, onde foram comparadas as respostas de 102 mães e 163 filhos face à resolução de conflitos, verificou que a perceção de uma resolução efetiva dos conflitos interparentais exerce um efeito positivo no ajustamento psicológico dos filhos. Também Whitton, Waldinger, Schulz, Allen, e Hauser (2008), com uma amostra de 43 indivíduos avaliados na adolescência e posteriormente na idade adulta, constatou que a forma como as figuras parentais resolvem os conflitos tende a replicar-se na maneira como os indivíduos resolvem os conflitos na idade adulta. Assim, sugere-se que as estratégias de resolução de conflitos utilizadas pelas figuras parentais se revelam extremamente significativas para os filhos, na medida em que podem funcionar como guias para a resolução de conflitos nas suas próprias relações de intimidade. Desta forma, uma vinculação segura às figuras primordiais pode contribuir para o bem-estar psicológico do indivíduo e, simultaneamente, para o desenvolvimento de relações posteriores pautadas por segurança. Experiências que ocorrem no sistema familiar, como a presença de conflitos interparentais não resolvidos, podem assumir-se como fatores de risco para a qualidade das relações primordiais e, concomitantemente, para o desenvolvimento de novas relações durante o restante processo vivencial. Como tal, torna-se premente aprofundar investigações neste âmbito, de forma a alcançar um conhecimento mais vasto e, assim, contribuir para intervenções mais precoces ao nível do impacto dos conflitos interparentais na vinculação às figuras primordiais e desenvolvimento de novas relações.

\section{Objetivos e hipóteses}

0 presente estudo teve como principal objetivo analisar a perceção de vinculação de adultos emergentes às suas figuras parentais, e testar o seu efeito na perceção da vinculação ao par amoroso. Pretendeu-se, ainda, testar o efeito mediador dos conflitos interparentais na associação entre a perceção da vinculação aos pais e a vinculação ao par amoroso. Espera-se que a dimensão de vinculação inibição da exploração e individualidade à mãe e ao pai se correlacione, positivamente com o evitamento e ambivalência, e os conflitos interparentais e, negativamente, com a confiança e dependência. Por outro lado, espera-se que as dimensões de vinculação relativas à qualidade do laço emocional e ansiedade de separação, à mãe e 
pai, se correlacionem positivamente com a vinculação amorosa (confiança e dependência), e negativamente com a vinculação amorosa (evitamento e ambivalência) e conflitos interparentais. Aguarda-se, ainda, que a qualidade do laço emocional a ambas as figuras parentais, enquanto variável positiva da dimensão da vinculação aos pais, prediga positivamente a vinculação ao par amoroso, nas dimensões de confiança e dependência. Por outro lado, espera-se que a qualidade do laço emocional prediga negativamente o evitamento e ambivalência na perceção de vinculação amorosa. Por último, espera-se que os conflitos interparentais exerçam um papel mediador na associação entre a vinculação aos pais e vinculação amorosa.

\section{MÉTODO}

\section{Participantes}

A amostra foi constituída por 505 sujeitos, 139 do sexo masculino e 366 do sexo feminino, com idades compreendidas entre os 18 e os 25 anos de idade $(M=20.59, D P=1.78)$. As habilitações literárias dos participantes variam entre o 9.o ano (3.o ciclo) e o ensino superior (mestrado) $(M=12.25, D P=.73$ ). Vivem com ambos os pais e irmãos 455 (89,4\%) dos jovens e apenas 12 (2,4\%) vivem com o namorado. Relativamente às relações amorosas, 277 (54.4\%) dos participantes mantêm atualmente um relacionamento amoroso, em média, há 29 meses $(M=29.10 ; D P=26.39)$, enquanto $232(45.6 \%)$ não mantêm atualmente qualquer relacionamento.

\section{Instrumentos}

Foi utilizado um Questionário de Dados Sociodemográficos, construído no sentido de abordar questões relativas às dimensões sociodemográficas (e.g. idade, sexo, escolaridade, com quem vive, há quanto tempo está numa relação) dos participantes.

Para a avaliação da perceção da vinculação aos pais, foi utilizado o Questionário de Vinculação ao Pai e à Mãe (QVPM) (Matos \& Costa, 2001, versão revista). Trata-se de um questionário de autorrelato que tem como objetivo aceder às representações de vinculação que os adolescentes e jovens adultos têm relativamente a cada uma das figuras parentais. Esta escala é composta por 30 itens, divididos em torno de uma estrutura de três fatores constituídos por dez itens cada: Inibição da Exploração e Individualidade (IEI, 10 itens) que visa avaliar a perceção de restrições à expressão da individualidade própria ("Os meus pais impõem a maneira deles de ver as coisas"); Qualidade do Laço Emocional (QLE, 10 itens), que pretende avaliar a importância das figuras parentais enquanto figuras de vinculação, percebidas como essenciais e insubstituíveis no desenvolvimento do indivíduo, a quem este recorrerá em situações de dificuldade e com as quais se desenvolve um sentido de continuidade relacional ("Confio nos meus pais para me apoiarem nos momentos difíceis da minha vida"); Ansiedade de Separação (AS, 10 itens) que aponta para uma experiência de ansiedade e de medo da separação das figuras de vinculação, reveladora de uma relação de dependência ("Só consigo enfrentar situações novas se os meus pais estiverem comigo"). Os itens estão apresentados numa escala do tipo Likert entre 1 ("Discordo totalmente") e 6 ("Concordo totalmente"). A análise de consistência interna demonstrou valores de alpha de Cronbach de .81 para o pai e .75 para a mãe relativamente à totalidade do instrumento. No que se refere à consistência interna de cada dimensão, registaram-se os seguintes valores de alpha Cronbach: $\mathrm{IEI}=.82 / .84, \mathrm{QLE}=.94 / .89$, AS = $.86 / .83$, para o pai e para a mãe respetivamente. As análises fatoriais confirmatórias indicaram bons valores de ajustamento para o modelo tanto para o pai, $\chi^{2}(22)=73.09, \chi^{2} / g l=3.32, p=.001$, CFI $=.983$, SRMR = .038, RMSEA = .068; como para a mãe: $\chi^{2}(22)=55.170, \chi^{2} / g l=2.51, p=.001, \mathrm{CFI}=.85$, SRMR $=$ $.032, \mathrm{RMSEA}=.054$.

Para medir a perceção da relação vinculação ao par amoroso foi utilizado o Questionário de Vinculação Amorosa (QVA) (versão reduzida de Matos, Barbosa, \& Costa, 2001). Esta escala é constituída por 25 itens, distribuídos por quatro dimensões: Confiança (6 itens) que procura avaliar a perceção do sujeito no que diz respeito à responsividade e à sensibilidade do companheiro para satisfazer as necessidades do indivíduo, isto é, em que medida o companheiro é percebido como fonte de conforto e apoio e constitui uma base segura de incentivo à exploração ("O(a) meu namorado(a) respeita os meus sentimentos"); Dependência (6 itens) que pretende avaliar a necessidade do indivíduo em procurar proximidade física e emocional, assim como a manifestação de ansiedade de separação e o medo de perder o par amoroso ("Fico muito nervoso(a) se não consigo encontrar a(o) minha (meu) namorada(o) quando preciso dela (ele)"); Evitamento (6 itens) que exprimem o facto de o companheiro ter um papel secundário nas suas necessidades de vinculação, dado que o sujeito se centra em si próprio e na sua competência para solucionar problemas ("Sei que sou capaz de resolver as coisas sozinho(a)") Ambivalência (7 itens) demonstram a insegurança do indivíduo, expressando irritabilidade perante situações inesperadas, e a dúvida do indivíduo relativamente ao seu papel enquanto figura amorosa assim 
como a dúvida relativamente às emoções sentidas face ao companheiro ("Gostava de ser a pessoa mais importante para ela (ele) mas não estou certo(a) de que assim seja"). Os itens estão apresentados numa escala do tipo Likert de seis pontos, que varia entre 1 ("Discordo totalmente") e 6 ("Concordo"). A análise de consistência interna demonstrou valor de alpha de Cronbach de .47 relativamente à totalidade do instrumento. Todavia, no presente estudo as dimensões do instrumento foram usadas separadamente, pelo que no que se refere à consistência interna de cada dimensão registaram-se valores de alpha de Cronbach de .90 para a Confiança, .82 para a Dependência, .71 para o Evitamento e .78 para a Ambivalência. A análise confirmatória apresentou valores ajustados para o modelo, $\chi^{2}(45)=149.209, \chi^{2}$ $/ g l=3.31, p=.001$, com CFI $=.97, \mathrm{RMR}=.04, \mathrm{RMSEA}=.07$.

Para avaliar a variável conflitos interparentais foi utilizado o Children's perception of interparental conflict scale (CPIC). Desenvolvido por Grych, Seid e Fincham (1992) e adaptado para uma população de adolescentes e jovens adultos da população portuguesa por Moura, Santos, e Matos (2006), este instrumento tem como objetivo avaliar a perceção que os adolescentes e jovens adultos têm relativamente à existência de conflitos entre as figuras parentais. Esta escala é constituída por 48 itens, divididos por três dimensões: Propriedades dos Conflitos interparentais; Culpa; e Ameaça. Na presente investigação, foi utilizada apenas a dimensão Propriedades dos Conflitos Interparentais, que pretende avaliar a perceção do jovem no que respeita à forma destrutiva dos conflitos interparentais. Esta dimensão engloba as escalas Frequência (6 itens), que coloca questões do tipo: "Eu vejo frequentemente os meus pais a discutirem", Intensidade (7 itens) que apresenta questões como: "Quando os meus pais têm uma discussão gritam muito um com o outro" e Resolução (6 itens) que procura avaliar em que medida o jovem perceciona os conflitos interparentais como não resolvidos: "Mesmo quando terminam uma discussão os meus pais continuam zangados" A resposta aos itens é efetuada numa escala tipo Likert entre 1 ("Discordo Totalmente") e 6 ("Concordo Totalmente"). De ressaltar que os itens 1, 2, 12, 18, 26, 27, 35 e 38 são invertidos. A análise de consistência interna demonstrou valor de alpha de Cronbach de .93 relativamente à totalidade do instrumento. No que respeita à consistência interna de cada dimensão, registaram-se valores de alpha de cronbach de .79 para Frequência, .89 para a Resolução e .84 para a Intensidade. As análises fatoriais confirmatórias apresentaram índices de ajustamento adequados, $\chi^{2}(20)$ $=92.074, \chi^{2} / g l=4.60, p=.001, \mathrm{CFI}=.98, \mathrm{SRMR}=.03, \mathrm{RMSEA}=.08$.

\section{Procedimento}

A recolha de dados foi realizada num momento único de forma transversal com jovens da Universidade de Trás-os-Montes e Alto Douro (UTAD), de acordo com um método de recolha probabilístico de forma aleatória simples, acrescendo elementos na população em geral da região norte do país, considerando apenas o enquadramento na faixa etária em estudo.

O protocolo foi previamente submetido ao Conselho de Ética da UTAD, tendo obtido um parecer positivo. Foi realizada uma reunião com os diretores de cada departamento no âmbito das várias escolas da UTAD, a quem foram solicitadas as devidas autorizações e clarificados diversos aspetos referentes ao estudo como a sua pertinência, estrutura e objetivos. Durante o preenchimento, a investigadora responsável encontrou-se presente de modo a esclarecer os objetivos gerais do estudo. Foi solicitado o consentimento dos participantes e dadas as instruções de preenchimento, sendo esclarecidas as dúvidas que os participantes apresentassem. Foram garantidos os pressupostos de voluntariedade, privacidade, anonimato e confidencialidade das informações prestadas.

0 tratamento dos dados foi realizado com o programa estatístico SPSS - Statistical Package for Social Sciences -, na sua versão 20.0 para o sistema Windows. Foram identificados e excluídos missings values (acima de 10\% de não respostas), e outliers procedendo-se ao cálculo da distância de Mahalanobis, optando-se por eliminar os índividuos que se desviavam significativamente da média geral de respostas (Field, 2005). Seguidamente, de forma a testar a estrutura original dos instrumentos utilizados, assim como os seus modelos teóricos propostos, procedeu-se à realização das Análises Confirmatórias de 1. ordem, através do programa estatístico EQS 6 - Structural Equations Program. De salientar que também foi calculado o valor do Alpha de Cronbach para a análise da consistência interna dos fatores. Para verificar se os dados da amostra seguiam os pressupostos de normalidade, procedeu-se à realização e interpretação do teste de Kolmogorov-Smirnov, bem como de gráficos de Histogramas, $Q-Q$ Plots, Scatterplots e Boxplots, uma vez que os mesmos fornecem informação acerca da distribuição dos dados (Maroco, 2007). Foram também analisados os valores de skeweness (assimetria) e kurtosis (achatamento), assumindo-se a normalidade sempre que os valores se encontrassem compreendidos no intervalo da sua unidade (-1 e 1) (Maroco, 2007). Os valores calculados confirmaram que a amostra em estudo cumpria os critérios de normalidade procedendo-se, desta forma, a análises estatísticas mediante testes paramétricos. No sentido de responder aos objetivos delineados para esta investigação, foi realizado um conjunto de análises estatísticas, nomeadamente análises correlacionais. Testou-se, ainda, a presença de 
um efeito mediador da variável conflitos interparentais na associação entre as variáveis qualidade do laço emocional aos pais e vinculação amorosa, através do Teste de Sobel (Baron \& Kenny, 1986).

\section{RESULTADOS}

A análise descritiva das variáveis em estudo permite observar que para a perceção da vinculação aos pais, as variáveis inibição da exploração e individualidade para pai e mãe apresentam níveis médios. Por outro lado, em ambas as figuras parentais verificam-se níveis elevados de qualidade do laço emocional e níveis médios altos de ansiedade de separação. Verificam-se ainda, relativamente à perceção da vinculação amorosa, elevados níveis de confiança e dependência, e níveis médios baixos de evitamento e ambivalência. No que concerne aos níveis de conflito, verificam-se na presente amostra níveis médios de frequência, intensidade e resolução (negativa) (Tabela 1).

Tabela 1. Correlação entre as variáveis, média e desvio-padrão (N=505).

\begin{tabular}{|c|c|c|c|c|c|c|c|c|c|c|c|c|c|}
\hline Variáveis & 1 & 2 & 3 & 4 & 5 & 6 & 7 & 8 & 9 & 10 & 11 & 12 & 13 \\
\hline \multicolumn{14}{|l|}{$\begin{array}{l}\text { Vinculação aos } \\
\text { pais: Pai }\end{array}$} \\
\hline $\begin{array}{l}\text { 1. Inibição da } \\
\text { exploração e } \\
\text { individualidade } \\
\text { 2. Qualidade do } \\
\text { laço emocional }\end{array}$ & $-.51^{* *}$ & - & & & & & & & & & & & \\
\hline $\begin{array}{l}\text { 3. Ansiedade de } \\
\text { separação }\end{array}$ & $-.01^{* *}$ & $.56^{* *}$ & - & & & & & & & & & & \\
\hline \multicolumn{14}{|l|}{$\begin{array}{l}\text { Vinculação aos } \\
\text { pais: Mãe }\end{array}$} \\
\hline $\begin{array}{l}\text { 4. Inibição da } \\
\text { exploração e } \\
\text { individualidade } \\
\text { 5. Qualidade do } \\
\text { laço emocional }\end{array}$ & -.40 & -.20 & .24 & $-.51^{* *}$ & - & & & & & & & & \\
\hline $\begin{array}{l}\text { 6. Ansiedade de } \\
\text { separação }\end{array}$ & .05 & .07 & .74 & $-.09^{* *}$ & $-.40^{* *}$ & - & & & & & & & \\
\hline \multicolumn{14}{|l|}{$\begin{array}{l}\text { Vinculação } \\
\text { Amorosa }\end{array}$} \\
\hline 7. Confiança & $-.23^{* *}$ & $.21^{* *}$ & $.10^{* *}$ & $-.23^{* *}$ & $.30^{* *}$ & $.13^{* *}$ & - & & & & & & \\
\hline 8. Dependência & $.12^{* *}$ & .06 & $.32^{*}$ & .08 & $.09^{* *}$ & $.34^{* *}$ & $.41^{* *}$ & - & & & & & \\
\hline 9. Evitamento & $.09^{* *}$ & $-.13^{* *}$ & $-.14^{* *}$ & $.09^{* *}$ & $-.17^{* *}$ & $-.15^{* *}$ & $-.55^{* *}$ & $-.52^{* *}$ & - & & & & \\
\hline 10. Ambivalência & $.26^{* *}$ & $-.09^{* *}$ & -.05 & $.28^{* *}$ & $-.11^{* *}$ & -.03 & $-.60^{* *}$ & $-.23^{* *}$ & $.49^{* *}$ & - & & & \\
\hline \multicolumn{14}{|l|}{$\begin{array}{l}\text { Conflitos } \\
\text { interparentais }\end{array}$} \\
\hline 11. Frequência & $.43^{* *}$ & $-.48^{* *}$ & $-.31^{* *}$ & $.31^{* *}$ & $-.27^{* *}$ & $-.09^{* *}$ & -.14 & .04 & .07 & .18 & - & & \\
\hline 12. Intensidade & $.39^{* *}$ & $-.41^{* *}$ & $-.23^{* *}$ & $.27^{* *}$ & $-.20^{* *}$ & -.05 & -.11 & .04 & .13 & .16 & $.79^{* *}$ & - & \\
\hline $\begin{array}{l}\text { 13. Resolução } \\
\text { (negativa) }\end{array}$ & $.39^{* *}$ & $-.57^{* *}$ & $-.33^{* *}$ & $.24^{* *}$ & $-.27^{* *}$ & $-.09^{* *}$ & -.21 & .05 & .08 & .18 & $.82^{* *}$ & $.70^{* *}$ & - \\
\hline$M$ & 2.75 & 5.14 & 3.69 & 2.80 & 5.51 & 3.93 & 5.10 & 3.40 & 2.19 & 2.44 & 2.97 & 2.75 & 2.90 \\
\hline$D P$ & .89 & .92 & .96 & .90 & .57 & .87 & .82 & 1.07 & .74 & .89 & 1.02 & .97 & 1.11 \\
\hline
\end{tabular}

$* p<0.05 ; * * p<.01$ 
Efeito da qualidade de vinculação aos pais na vinculação amorosa: papel mediador dos conflitos interparentais

De modo a analisar o papel mediador dos conflitos interparentais, foram tidas em consideração as relações entre as variáveis, nomeadamente, da qualidade de vinculação ao pai e à mãe como preditoras quer da vinculação amorosa (confiança, dependência, evitamento e ambivalência) quer dos conflitos interparentais (frequência, resolução e intensidade). Da escala da qualidade de vinculação ao pai e à mãe, foi utilizada apenas a dimensão qualidade do laço emocional enquanto dimensão positiva da qualidade da vinculação que melhor se adequa aos objetivos do estudo.

Assim, a análise de equações estruturais permitiu observar um efeito inicial positivo da qualidade de laço emocional ao pai no desenvolvimento de confiança e dependência $(\beta=.24)$ e negativo no desenvolvimento de evitamento e ambivalência $(\beta=-.56)$. De igual modo, foi possível verificar que a qualidade de laço emocional ao pai denota uma predição negativa nos conflitos interparentais $(\beta=-.28)$, assim como se verificou um efeito negativo dos conflitos interparentais no desenvolvimento de confiança e dependência $(\beta=-.28)$ e positivo no desenvolvimento de evitamento e ambivalência $(\beta=.91)$.

No modelo final, após introduzida a variável mediadora dos conflitos interparentais, atestou-se que o efeito inicial direto da qualidade de laço emocional ao pai sobre a confiança e dependência $(\beta=.24)$ diminuiu $(\beta=.15)$, observando-se uma mediação parcial (Sobel test $z=1.59, S E=.03 p<.001, \beta=.047$ ). Por outro lado, os resultados mostram que o efeito inicial direto da qualidade do laço emocional sobre o evitamento e ambivalência $(\beta=-.56)$ perdeu significância $(\beta=-.07)$, verificando-se deste modo uma mediação total dos conflitos interparentais (Sobel test $z=-14.25, S E=.01, p<.001 \beta=-.131$ ). No que concerne aos índices de ajustamento, estes encontram-se adequados $\chi^{2}(24)=125.5, \chi^{2} / g l=5.22, p=.001$, $\mathrm{CFI}=.96 ; \mathrm{SRMR}=.04 ; \mathrm{RMSEA}=.09$.

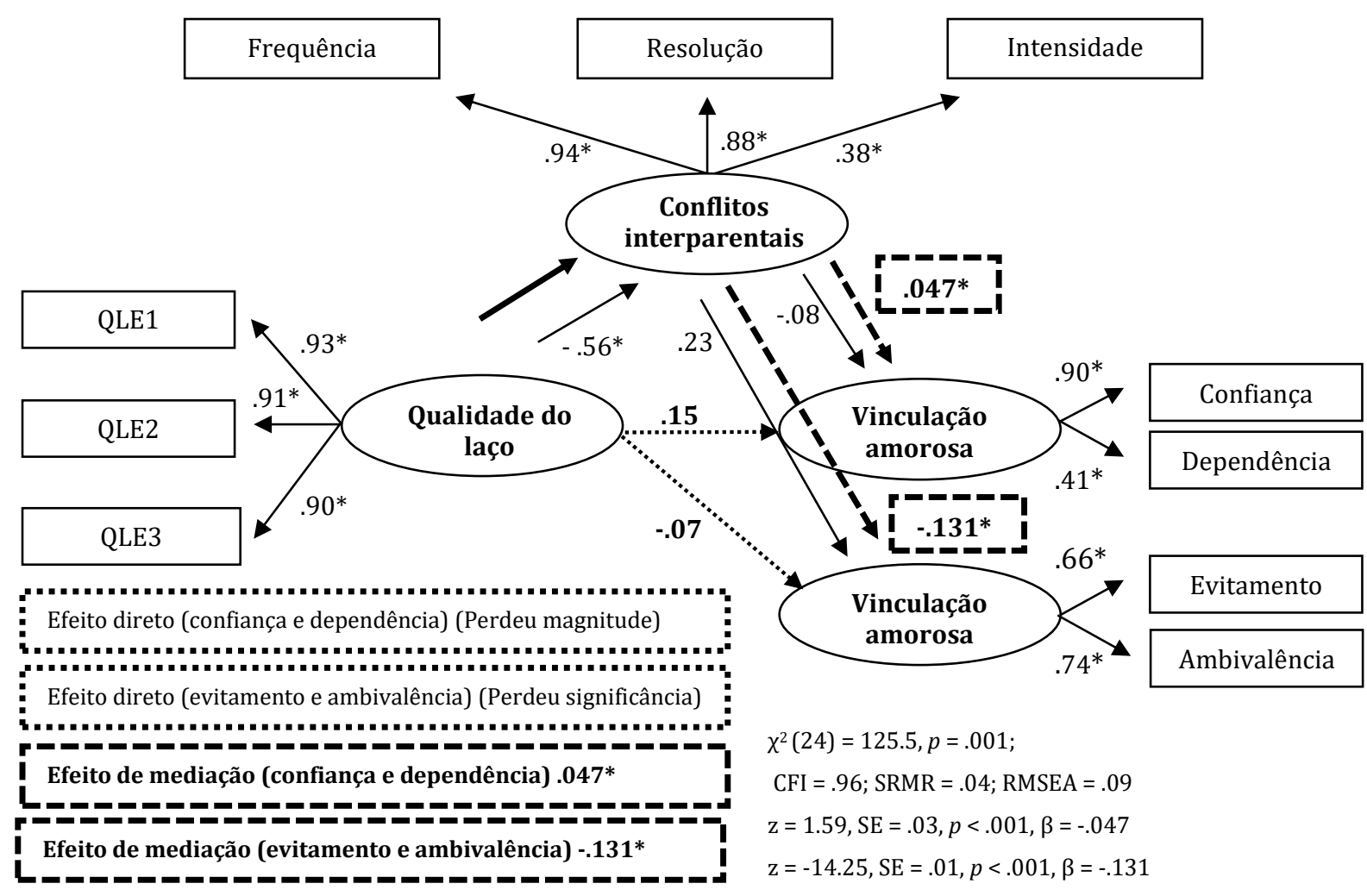

Figura 1. Modelo representativo do efeito mediador dos conflitos interparentais na associação entre qualidade do laço emocional ao pai e vinculação amorosa 
Tabela 1. Passos do Teste de Sobel para o efeito mediador dos conflitos interparentais na associação entre qualidade do laço emocional ao pai e vinculação amorosa.

\begin{tabular}{|c|c|c|c|c|}
\hline $\begin{array}{l}\text { Passos do Teste de Sobel } \\
\text { Modelo Pai }\end{array}$ & B & SE & $\beta$ & $P$ \\
\hline $\begin{array}{l}\text { 1.- Passo - Qualidade do laço emocional- Confiança e } \\
\text { Dependência }\end{array}$ & .32 & .00 & .24 & $<.001$ \\
\hline $\begin{array}{l}\text { 1. } \mathrm{o} \text { Passo - Qualidade do laço emocional - Evitamento e } \\
\text { Ambivalência }\end{array}$ & -.09 & .03 & -.16 & $<.001$ \\
\hline $\begin{array}{l}\text { 2. o Passo - Qualidade do laço emocional - Conflitos } \\
\text { interparentais }\end{array}$ & -.57 & .04 & -.56 & $<.001$ \\
\hline $\begin{array}{l}\text { 3.o Passo - Conflitos interparentais - Confiança e } \\
\text { Dependência (QVA) }\end{array}$ & -.08 & .05 & -.15 & $<.001$ \\
\hline $\begin{array}{l}\text { 3. - Passo - Conflitos interparentais - Evitamento e } \\
\text { Ambivalência (QVA) }\end{array}$ & .09 & .00 & .19 & $<.001$ \\
\hline $\begin{array}{l}\text { 4. - Passo - Qualidade do Laço Emocional - Conflitos } \\
\text { interparentais - Confiança e Dependência }\end{array}$ & .070 & .043 & .047 & $<.001$ \\
\hline $\begin{array}{l}\text { 4. - Passo - Qualidade do Laço Emocional - Conflitos } \\
\text { interparentais - Evitamento e Ambivalência }\end{array}$ & -.109 & -.009 & -.131 & $<.001$ \\
\hline
\end{tabular}

Relativamente à figura materna, a testagem do modelo verificou que inicialmente a variável qualidade do laço emocional à mãe assumiu um efeito positivo sobre a confiança e dependência $(\beta=.35)$ e negativo sobre o evitamento e ambivalência $(\beta=-.18)$. Constatou-se ainda que a qualidade de laço emocional à mãe prediz negativamente os conflitos interparentais $(\beta=-.28)$, assim como se observou um efeito negativo dos conflitos interparentais na confiança e dependência $(\beta=-.15)$ e positivo no evitamento e ambivalência $(\beta=.19)$. Quando se introduziu a variável mediadora dos conflitos interparentais na equação final, atestou-se que o efeito direto inicial da qualidade do laço emocional à mãe sobre a confiança e dependência $(\beta=.35)$ diminui $(\beta=.22)$, denotando-se uma mediação parcial (Sobel test $\mathrm{z}=$ $1.54 \mathrm{SE}=.02, p=.001 \beta=.029$ ).

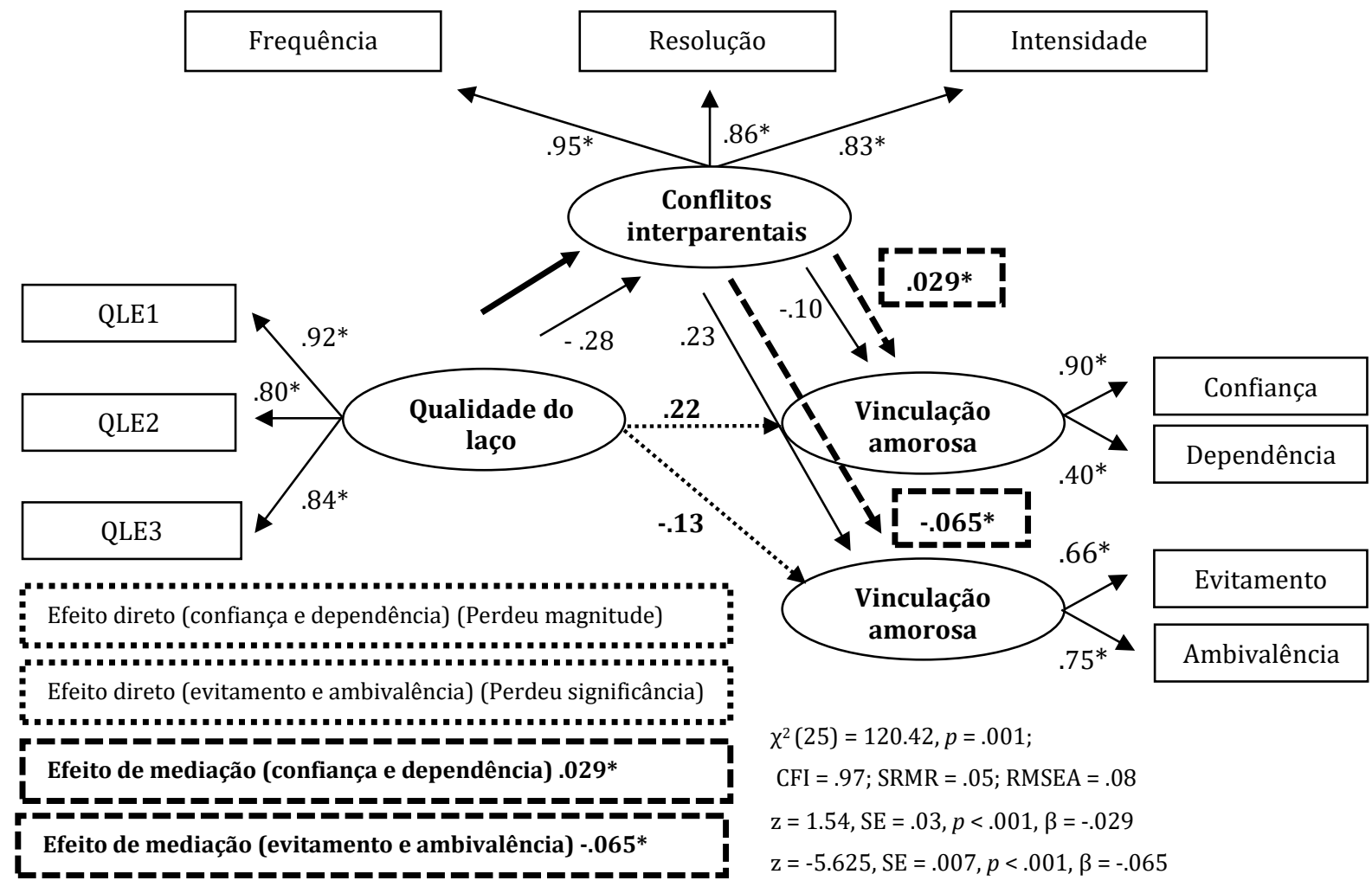

Figura 2. Modelo representativo do efeito mediador dos conflitos interparentais na associação entre qualidade do laço emocional à mãe e vinculação amorosa 
Por outro lado, o efeito direto inicial sobre o evitamento e ambivalência $(\beta=-.18)$ perde significância $(\beta=-.13)$, apontando para uma mediação total dos conflitos interparentais (Sobel test $z=$ $5.625 \mathrm{SE}=.007, p=.001, \beta=-.065)$. Os índices de ajustamento encontram-se adequados $\chi^{2}(25)=120.42$, $\chi^{2} / g l=4.81, p=.001, \mathrm{CFI}=.97, \mathrm{SRMR}=.05, \mathrm{RMSEA}=.08$.

Tabela 2. Passos do Teste de Sobel para o efeito mediador dos conflitos interparentais na associação entre qualidade do laço emocional à mãe e vinculação amorosa.

\begin{tabular}{|c|c|c|c|c|}
\hline $\begin{array}{l}\text { Passos do Teste de Sobel } \\
\text { Modelo Mãe }\end{array}$ & B & SE & $\beta$ & $P$ \\
\hline $\begin{array}{l}\text { 1.- Passo - Qualidade do laço emocional- Confiança e } \\
\text { Dependência }\end{array}$ & .24 & .00 & .35 & $<.001$ \\
\hline $\begin{array}{l}\text { 1.- Passo - Qualidade do laço emocional - Evitamento e } \\
\text { Ambivalência }\end{array}$ & -.21 & .00 & -.18 & $<.001$ \\
\hline $\begin{array}{l}\text { 2. o Passo - Qualidade do laço emocional - Conflitos } \\
\text { interparentais }\end{array}$ & -.45 & .08 & -.28 & $<.001$ \\
\hline $\begin{array}{l}\text { 3. Passo - Conflitos interparentais - Confiança e } \\
\text { Dependência (QVA) }\end{array}$ & -.08 & .05 & -.15 & $<.001$ \\
\hline $\begin{array}{l}\text { 3.․ Passo - Conflitos interparentais - Evitamento e } \\
\text { Ambivalência (QVA) }\end{array}$ & .09 & .00 & .19 & $<.001$ \\
\hline $\begin{array}{l}\text { 4. }{ }^{\circ} \text { Passo - Qualidade do Laço Emocional - Conflitos } \\
\text { interparentais - Confiança e Dependência }\end{array}$ & .046 & .008 & .029 & $<.001$ \\
\hline $\begin{array}{l}\text { 4.- Passo - Qualidade do Laço Emocional - Conflitos } \\
\text { interparentais - Evitamento e Ambivalência }\end{array}$ & -.090 & .026 & -.065 & $<.001$ \\
\hline
\end{tabular}

\section{DISCUSSÃo}

O desenvolvimento das relações seguras com as figuras parentais sugere ser preditor de maior disponibilidade para o estabelecimento de relações saudáveis por parte dos jovens emergentes com o seu par amoroso (Mikulincer et al., 2002; Morgan \& Shaver, 1999). O presente estudo teve como principal objetivo analisar as associações entre da perceção da qualidade de vinculação aos pais sobre a qualidade de vinculação ao par amoroso em jovens adultos, assim como testar o papel mediador dos conflitos interparentais na associação anterior.

Os resultados observados sugerem que a inibição da exploração e individualidade se associa positivamente com o evitamento, ambivalência e dependência e negativamente com a confiança. Por sua vez, a qualidade do laço emocional associa-se de forma positiva com a confiança e dependência e de forma negativa com o evitamento e ambivalência. Evidências empíricas demonstram que uma vinculação segura às figuras significativas é preditora do desenvolvimento saudável de relações românticas na vida adulta, sendo estas relações caracterizadas pela confiança, satisfação, interdependência e suporte mútuo. Porém, quando as relações com as figuras primordiais são tidas como pouco apoiantes, os sujeitos tendem a estabelecer relações mais instáveis e ambivalentes com o par romântico (e.g. Beato, 2008; Fraley, 2002; Melo \& Mota, 2014; Meier, Carr, Currier, \& Neimeyer, 2013; Pereira et al., 2012; Waters \& Cummings, 2000). Os resultados do estudo de Altin e Terzi (2010) corroboram esta ideia ao referir que indivíduos com uma vinculação segura às figuras primordiais tendem a desenvolver relações amorosas de maior segurança, enquanto que os mais inseguros tendem a ser mais dependentes, estabelecendo relacionamentos com baixos níveis de confiança, satisfação e compromisso.

Neste estudo, indivíduos que percecionam conflitos interparentais não resolvidos denotam uma maior inibição da exploração e individualidade a ambas as figuras parentais e, concomitantemente, apresentam menor qualidade de laço emocional aos pais. Denotam, ainda, maior ansiedade de separação relativamente à figura paterna. Neste sentido, e de acordo com os resultados, sugere-se que a existência de um ambiente familiar caracterizado por relações conflituosas entre o casal pode afetar a relação entre pais e filhos, comprometendo a qualidade da vinculação, questão que assume particular relevância face à figura paterna. Mota e Matos (2014) referem num estudo realizado com 584 adultos emergentes que as relações com os pais pautadas pela satisfação, intimidade e afeto se associam negativamente com o desenvolvimento de conflitos interparentais e ao aumento de auto-estima e diminuição de sintomatologia depressiva. Correia e Mota (2016) sugerem o papel do ambiente familiar com coesão, expressividade entre os membros e menores níveis de conflito, no desenvolvimento positivo das relações românticas em 432 adultos emergentes. De acordo com a literatura, o conflito destrutivo prejudica a qualidade das relações de vinculação pais-filhos, uma vez que aumenta o número de interações negativas e diminui o envolvimento e disponibilidade emocional dos pais (Buchanan, \& Waizenhofer, 2001; Grych et al., 1992; Moura et al., 2010). Deste modo, o conflito parental parece ser um aspeto importante nas relações 
familiares, uma vez que se manifesta como uma variável capaz de comprometer o estabelecimento de um vínculo seguro aos pais (Mota \& Matos, 2014).

Por último, e de acordo com os objetivos propostos, os resultados apontam ainda para o papel preditor da qualidade da vinculação aos pais na qualidade da vinculação amorosa nos jovens, tendo-se observado, tal como esperado, uma predição positiva sobre a confiança e dependência, e negativa sobre o evitamento e ambivalência. Estes resultados são consistentes com a perspetiva de Bowlby (1969), que salienta que a existência de um vínculo caracterizado por amor e apoio com as figuras primordiais de afeto potencia a co-construção de modelos internos dinâmicos positivos de si e dos demais. Evidências empíricas apontam que uma vinculação segura às figuras significativas é preditora do desenvolvimento saudável de relações românticas na vida adulta, sendo estas relações caracterizadas pela confiança, satisfação, interdependência e suporte mútuo. Por outro lado, quando as relações com as figuras primordiais são tidas como pouco apoiantes, os sujeitos tendem a estabelecer relações de vinculação inseguras com o par romântico, pautadas por baixos níveis de confiança, satisfação e compromisso (e.g. Altin \& Terzi, 2010; Beato, 2008; Fraley, 2002; Machado, 2007; Pereira et al., 2012; Waters \& Cummings, 2000). Deste modo, os resultados vão ao encontro da ideia original de Bowlby (1988), pelo que a qualidade da relação que se desenvolve entre pais e filhos mostra-se significativa, no sentido em que permite criar bases seguras que potenciem maior satisfação e estabilidade nas relações futuras, nomeadamente de cariz romântico.

Ainda nesta análise, destaca-se o papel dos conflitos interparentais enquanto variável mediadora na associação entre qualidade de vinculação aos pais e vinculação amorosa. Neste sentido, os conflitos interparentais apresentaram um efeito mediador na medida em que a qualidade do laço emocional aos pais parece partilhar o seu efeito preditor na qualidade da relação romântica com os conflitos interparentais. Tal como seria esperado, verificou-se que a vinculação com os pais se associa negativamente com conflitos interparentais não resolvidos com elevada intensidade e frequência, o que por sua vez se associa a uma ligação com o par amoroso mais pautada por ambivalência e evitamento, e menos confiança. Nesta medida, apesar da qualidade da vinculação aos pais assumir um papel significativo na qualidade da relação amorosa, os conflitos interparentais podem constituir um precipitante de maiores dificuldades na qualidade da relação amorosa dos jovens (Melo \& Mota, 2014). Vários estudos realizados neste âmbito, com o objetivo de verificar o efeito da variável conflitos interparentais nas relações românticas de jovens adultos, atestaram um impacto negativo do conflito interparental na qualidade e manutenção de relações românticas (e.g., Cui \& Fincham, 2010; Cusimano \& Riggs, 2013; Dinero, Conger, Shaver, Widaman, \& Larsen-Rife, 2008; Feeney, 2006). Do acordo com Herzog e Coney (2002), os padrões destrutivos de comportamentos observados no seio familiar, como os conflitos interparentais, podem ser reproduzidos com figuras significativas de afeto fora do seio familiar, ameaçando o bem-estar emocional do par romântico, bem como a qualidade dos padrões comunicacionais estabelecidos entre ambos. Beato (2008) reforça esta ideia, salientando que a perceção de frequentes e intensos conflitos interparentais leva a que estes assumam um padrão relacional inseguro, que consequentemente se repercute nas relações românticas, podendo ocasionar menor confiança no parceiro amoroso.

\section{Implicações práticas e limitações}

0 presente estudo assume um contributo positivo na compreensão da importância da qualidade de vinculação aos pais no desenvolvimento de relações amorosas satisfatórias, e o impacto que as vivências de conflitos interparentais acarretam. Neste sentido, o estudo vem ao encontro de um conjunto de evidências já existentes e que sublinham a necessidade de promover o estabelecimento de relações saudáveis entre os pais e as crianças, no sentido de contribuir para a qualidade das relações na vida adulta. Nesta medida, seria oportuno desenvolver programas de intervenção e prevenção precoces por forma a minimizar as consequências negativas advindas dos conflitos interparentais, assim como desenvolver estratégias de coping para lidar com as adversidades e prevenir o desenvolvimento de sintomatologia psicopatológica associada.

Acrescem-se ao presente estudo algumas limitações, nomeadamente, o recurso a instrumentos de autorrelato passíveis de enviesamentos, e o facto da investigação ser de cariz transversal, não possibilitando analisar a comparação dos resultados ao longo do tempo, nem o estabelecimento de relações de causalidade. Ainda neste contexto, salientam-se algumas características intrínsecas da amostra, especificamente o facto de ser maioritariamente composta por estudantes universitários.

Em termos de recomendações de investigação futuras, seria importante contemplar outras variáveis sociodemográficas como a configuração familiar, variáveis relacionais como a qualidade da vinculação aos irmãos e aos pares (testando o seu efeito protetor) e ainda a inclusão de outras variáveis psicossociais e desenvolvimentais (competências sociais, resiliência, coping, entre outros). Poderia ainda 
propor-se a inclusão de uma análise multinformante (por parte dos jovens e dos pais). Seria igualmente relevante optar por um estudo de caráter longitudinal, incluir análises qualitativas como entrevistas semiestruturadas com os participantes e contemplar uma faixa etária mais abrangente.

\section{REFERÊNCIAS}

Ainsworth, M. (1969). Object relations, dependency, and attachment: Theoretical review of the infantmother relationship. Child Development, 40, 969-1026.

Ainsworth, M. (1991). Attachments and other affectional bonds across the life cycle. In C.M. Parkes, J. Hinde-Stevenson \& P. Marris. (Ed), Attachment across the life cycle (32-49). London- Routledge.

Altin, M., \& Terzi, S. (2010). How does attachment styles relate to intimate relationship to aggravate the depressive symptoms? Procedia Social and Behavioral Sciences, 2, 1008-1015. http://dx.doi.org/10.1016/j.sbspro.2010.03.142

Atger, F. (2004). Vinculação e adolescência. In N. Guedeney \& A. Guedeney (Ed), Vinculação: Conceitos e aplicações (pp.147-153). Lisboa: Climepsi.

Baron, M., \& Kenny, A. (1986). The moderator-mediator variable distinction in social psychological research: Conceptual, strategic and statistical considerations. Journal of Personality and Social Psychology, 51, 1173-1182.

Beato, A. (2008). "Adolescer" entre relações. Parentalidade, amizade e amorosidade: Que contributos na transição para a idade adulta? (Dissertação de mestrado não publicada). Faculdade de Psicologia e Ciências da Educação, Universidade de Lisboa.

Benetti, S. (2006). Conflito conjugal: Impacto no desenvolvimento psicológico da criança e do adolescente. Psicologia: Reflexão e Crítica, 19(2), 261-268. http://dx.doi.org/10.1590/S010279722006000200012

Bowlby, J. (1969). Attachment and loss.Vol.1: Attachment. New York: Basic Books.

Bowlby, J. (1988). A secure base: Parent-child attachment and healthy human development. London: Basic Books.

Bretherton, I. (1985). Attachment theory: Retrospect and prospect. In I. Bretherton \& E. Waters (Eds.), Growing points of attachment theory and research. Monographs of the Society for Research in Child Development, 50(1-2, Serial No. 209), 3-35.

Bretherton, I. (2000). The origins of attachment theory: John Bowlby and Mary Ainsworth. In Goldberg, S., Muir, R., \& Herr, J.; Attachment theory: social, developmental, and clinical perspectives. London: The Analitic Press.

Buchanan, C. M., \& Waizenhofer, R. (2001). The impact of interparental conflict on adolescent children: Considerations of family systems and family structure. En A. Booth, A. C. Crouter \& M. Clements (Eds.), Couples in conflict (pp. 149-160). Mahwah, NJ: Erlbaum.

Castillo, M. (2007). Los padres y los hijos: Variables de riesgo. Educación y Educadores, 10(1), 27-37.

Correia, F., \& Mota, C.P. (2016). Ambiente familiar e qualidade da vinculação amorosa: papel mediador da individuação em jovens adultos. Análise Psicológica, 34(1), 15-29. https://doi.org/10.14417/ap.1018

Crowell, J. A., Treboux, D., \& Waters, E. (2002). Stability of attachment representations: The transition to marriage. Developmental Psychology, 38, 467-479.

Cui, M., \& Fincham, D. (2010). The differential effects of parental divorce and marital conflict on young adult romantic relationships. Personal Relationships, 17, 331-343. https://doi.org/10.1111/j.14756811.2010.01279.x

Cummings, E. M. (1998). Children exposed to marital conflict and violence: Conceptual and theoretical directions. In G. Holden, B. Geffner \& E. Jouriles (Eds.), Children exposed to marital violence: Theory, research, and applied issues (pp. 21-53). Washington, DC: American Psychological Association.

Cummings, E.M., Goeck-Morey, M.C., \& Papp, L.M. (2004). Everyday marital conflict and child aggression. $\begin{array}{llll}\text { Journal of Abnormal Child Psychology, 32(2), } & \text { 191-202. }\end{array}$ https://doi.org/10.1023/B:JACP.0000019770.13216.be

Cusimano, A., \& Riggs, S. (2013). Perceptions of interparental conflict, romantic attachment, and psychological distress in college students. Couple and Family Psychology: Research and Practice, 2(1), 45-59. http://dx.doi.org/10.1037/a0031657

Dinero, R., Conger, R., Shaver, P., Widaman, K., \& Larsen-Rife, D. (2008). Influence of family of origin and adult romantic partners on romantic attachment security. Journal of Family Psychology, 22(3), 622632. https://doi.org/10.1037/a0012506

Feeney, C., Cassidy, J., \& Ramos-Marcuse, F. (2008). The generalization of attachment representations to new social situations: predicting behavior during initial interactions with strangers. Jornal of Personality and Social Psychology, 95(6), 1481-98. https://doi.org/10.1037/a0012635 
Feeney, J. (2006). Parental attachment and conflict behavior: Implications for offspring's attachment, loneliness, and relationship satisfaction. Personal Relationships, 13(1), 19-36. https://doi.org/10.1111/j.1475-6811.2006.00102.x

Field, A. (2005). Discovering statistics using SPSS. London: Sage Publications.

Fraley, R. (2002). Attachment stability from infancy to adulthood: Meta-analysis and dynamic modeling of developmental mechanisms. Personality and Social Psychology Review, 6(2), 123-151.

García, V., Marín, I., \& Currea, F. (2006). Relaciones maritales, relaciones paternas y su influencia en el ajuste psicológico de los hijos. Acta Colombiana de Psicologia, 9(2), 115-126.

Goeke-Morey, M., Cummings, E., \& Papp, L. (2007). Children and marital conflict resolution: Implications for emotional security and adjustment. Journal of Family Psychology, 21(4), 744-753.

Grych, J. H., \& Fincham, F. D. (1990). Marital conflict and children's adjustment: A cognitive-contextual framework. Psychological Bulletin, 108, 267-90.

Grych, J. H., Seid, M., \& Fincham, F. D. (1992). Assessing marital conflict from the child's perspective: The children's perception of interparental conflict scale. Child Development, 63(3), 558-572.

Hazan, C., \& Shaver, P. R. (1990). Love and work: an attachment-based theoretical perspective. Journal of Personality and Social Psychology, 52, 511-524.

Herzog, M., \& Cooney, T. (2002). Parental divorce and perceptions of past interparental conflict: Influences on the communication of young adults. Journal of Divorce \& Remarriage, 36(34), 89-109. https://doi.org/10.1300/J087v36n03_06

Lindsey, W., Colwell, J., Frabutt, M., \& MacKinnon-Lewis, C. (2006). Family conflict in divorced and nondivorced families: Potential consequences for boy's friedship status and friendship quality. Journal of Social and Personal Relationships, 23, 45-63. https://doi.org/10.1177/0265407506060177

Machado, S. (2007). Padrões de vinculação aos pais em adolescentes e jovens adultos e adaptação à Universidade. Revista Portuguesa de Pedagogia, 41(2), 5-28.

Maroco, J. (2007). Análise estatística com utilização do SPSS. Lisboa: Sílabo.

Matos, M. (2002). (Des)continuidades na vinculação aos pais e ao par amoroso em adolescentes. (Tese de Doutoramento): Faculdade de Psicologia, Instituto de Consulta Psicológica, Formação e Desenvolvimento, Universidade do Porto.

Matos, M., \& Costa, E. (2006). Vinculação aos pais e ao par romântico em adolescentes. Psicologia, 20(1), 97-126.

Matos, P., Barbosa, S., \& Costa, M. (2001). Avaliação da vinculação amorosa em adolescentes e jovens adultos: Construção de um instrumento e estudos de validação. Revista Iberoamericana de Diagnóstico y Evaluación Psicológica, 11(1), 93-109.

Matos, P., \& Costa, M. (2001). Questionário de vinculação ao pai e à mãe. Manuscrito não publicado. Faculdade de Psicologia e de Ciências da Educação da Universidade do Porto.

Melo, O., \& Mota, C. (2014). Interparental conflicts and the development of psychopathology in adolescents and young adults. Paidéia, 24(59), 283-293. http://dx.doi.org/10.1590/198243272459201402

Meier, M., Carr, R., Currie, M., \& Neimeyer, A. (2013). Attachment anxiety and avoidance in coping with bereavement: Two studies. Journal of Social and Clinical Psychology, 32(3), 315-334. https://doi.org/10.1521/jscp.2013.32.3.315

Meeus, W., Iedema, J., Maassen, G., \& Engels, R. (2005). Separation-individuation revisited: On the interplay of parent-adolescent relations, identity and emotional adjustment in adolescence. Journal of Adolescence, 28, 89-106. https://doi.org/10.1016/j.adolescence.2004.07.003

Michael, K., \& Ben-Zur, H. (2007). Risk-taking among adolescents: association with social and affective factors. Journal of Adolescence, 30, 17-31. https://doi.org/10.1016/j.adolescence.2005.03.009

Mikulincer, M., Gillath, O., \& Shaver, P. R. (2002). Activation of the attachment system in adulthood: threat-related primes increase the accessibility of mental representations of attachment figures. Journal of Personality and Social Psychology, 83, 881-895. https://doi.org/10.1037/00223514.83.4.881

Monteiro, L., Veríssimo, M., Vaughn, E., Santos, J., \& Fernandes, M. (2008). Análise do fenómeno de base segura em contexto familiar: As relações criança/mãe e criança/pai. Psicologia, 22(1), 104-25.

Mota, C., \& Matos, P. (2009). Clinical implications of interparental conflict: Intervention with adolescents from different family contexts. Comunicação apresentada no 40th Society for Psychotherapy Research (SPR) International Meeting. Santiago do Chile, Chile.

Mota, C., \& Matos, P. (2011). Adolescência e conflitos parentais: Uma perspectiva de resiliência. In P.M. Matos, C. Duarte, \& M.E. Costa (Coords.). Famílias: Questões de desenvolvimento e intervenção (p.125-251). Porto: LivPsic. 
Mota, C. \& Matos, P. (2014). Relación parental, autoestima y sintomatología depresiva en jóvenes adultos. Implicaciones de los conflictos interparentales, coalición y triangulación. Universitas Psychologica, 13(3), 907-922. http://dx.doi.org/10.11144/Javeriana.UPSY13-3.rpas

Moura, O., \& Matos, P. (2008).Vinculação aos pais, divórcio e conflito interparental em adolescentes. Psicologia, 22(1), 127-152.

Moura, O., Santos, R., \& Matos, M. (2006). Children`s perception of interparental conflict scale: Análise factorial confirmatória com adolescentes e jovens adultos. XI Conferência Internacional - Avaliação psicológica: Formas e contextos - 5, 6 e 7 de Outubro, Universidade do Minho.

Moura, O., Santos, R. A., Rocha, M., \& Matos, P. M. (2010). Children's Perception of Interparental Conflict Scale (CPIC): Factor structure and invariance across adolescents and emerging adults. $\begin{array}{lllll}\text { International of Journal } & \text { 364ting, }\end{array}$ http://dx.doi.org/10.1080/15305058.2010.487964

Morgan, M., \& Shaver, R. (1999). Attachment processes and commitment to romantic relationships. In J. M. Adams \& H. Jones (Eds.), handbook of interpersonal commitment and relationship stability (pp. 109-124). New York: Kluwer Academic.

Pereira, M., Ferreira, G., \& Paredes, A. (2012). Apego aos pais, relações românticas, estilo de vida, saúde física e mental em universitários. Psicologia: Reflexão e Crítica, 26(4), 762-771. http://dx.doi.org/10.1590/S0102-79722013000400017

Reese-Weber, M., \& Kahn, H. (2005). Familial predictors of sibling and romantic-partner conflict resolution: comparing late adolescents from intact and divorced families. Journal of Adolescence, 28(4), 479-493. http://dx.doi.org/10.1016/j.adolescence.2004.09.004

Rocha, M., Mota, C. P., \& Matos, P. M. (2011). Vinculação à mãe e ligação aos pares na adolescência: 0 papel mediador da auto-estima. Análise Psicológica, 2, 185-200. http://dx.doi.org/10.14417/ap.47

Shaver, P. R., \& Hazan, C. (1988). A biased overview of the study of love. Journal of Social and Personal Relationships, 5, 473-510.

Treboux, D., Crowell, J., \& Waters, E. (2004). When "new" meets "old": Configurations of adult attachment representations and their implications for marital functioning. Developmental Psychology, 40, 295314. http://dx.doi.org/10.1037/0012-1649.40.2.295

Waters, E., \& Cummings, E. (2000). A secure base from which to explore close relationships. Child Development, 71, 1-13. http://dx.doi.org/10.1111/1467-8624.00130

Waters, H., Waters, E., \& Crowell, J. (2000). The attachment narratives studies. Unpublished manuscript, State University of New York at Stony Brook.

Whitton, W., Waldinger, R., Schulz, M., Allen, J., \& Hauser, S. (2008). Prospective associations from familyof-origin interactions to adult marital interactions and relationship adjustment. Journal of Family Psychology, 22(2), 274-286. https://doi.org/10.1037/0893-3200.22.2.274.

Zimmermann, P. (2004). Attachment representations and characteristics of friendship relations during adolescence. Journal of Experimental Child Psychology, 88, 83-101. http://dx.doi.org/10.1016/j.jecp.2004.02.002

Historial do artigo

Recebido 10/08/2016

Aceite $\quad 02 / 10 / 2017$

Publicado 05/2018 
Vinculação, relação amorosa e conflitos interparentais

Esta página encontra-se propositadamente em branco 